\title{
CNN Technology for Spatiotemporal Signal Processing
}

\author{
David López Vilariño, ${ }^{1}$ Diego Cabello Ferrer, ${ }^{1}$ Víctor Manuel Brea Sánchez, ${ }^{1}$ \\ Ronald Tetzlaff, ${ }^{2}$ and Chin-Teng Lin $^{3}$
}

${ }^{1}$ University of Santiago de Compostela, Spain

${ }^{2}$ Dresden University of Technology, Germany

${ }^{3}$ National Chiao-Tung University (NCTU), Hsinchu, Taiwan

Correspondence should be addressed to David López Vilariño, david.vilarino@usc.es

Received 18 October 2009; Accepted 18 October 2009

Copyright (c) 2009 David López Vilariño et al. This is an open access article distributed under the Creative Commons Attribution License, which permits unrestricted use, distribution, and reproduction in any medium, provided the original work is properly cited.

Cellular Neural Networks (CNNs) are a paradigm for nonlinear spatial-temporal dynamics and the core of the Cellular Wave Computing (also called CNN technology). Partial Differential Equations (PDE) or wave-like phenomena are the computing primitives of CNN. Besides, their suitability for physical implementation makes CNNs very appropriate for high-speed parallel signal processing.

Since its inception by Professor Chua in 1988, who defined a CNN as an arrangement of regularly spaced cells which usually communicate with each other through their nearest neighbors [1], many advances in theory, applications and implementation have emerged. Early CNN applications were mainly focused on image processing. The availability of cellular processor arrays with a high number of processing elements paved the way for the development of new applications and the recovery of techniques traditionally conditioned by the slow speed of conventional computers. Let us name, as example, image processing techniques based on active wave propagation, or applications within the medical image processing framework, where fast processing provides new capabilities for medical disease diagnosis. Also, emerging applications exploit the complex spatiotemporal phenomena exhibited by multilayer CNN and extend to the modeling of neural circuits for biological vision, motion, and higher brain function.

Besides, today one of the most decisive factors in CNN research is the appearance of many new exciting technological advances. The impressive pace of the Moore's Law has led to the introduction of ever more complex CMOS digital approaches like high-end FPGAs, multicore general purpose microprocessors, or general purpose Graphic Processing
Units (GPU). All these platforms offer the performance of ASIC chips from the recent past, allowing for the rapid development of not only proof-of-concept or prototype approaches but also final solutions. At the same time, advances like CMOS-3D technology or new nanodevices fuel the design of custom analog or mixed-signal CNNs. All of these implementations are interwoven with the progress in theory and applications. The nine papers collected on this Special Issue gather advances in theory, applications and, implementation, giving a snapshot of the state-of-the-art of the $\mathrm{CNN}$ technology.

The first two papers of this Special Issue by Mária ErcseyRavasz et al. and by Mauro Di Marco et al. are placed midway between theory and applications. The first paper touches on a very well-known problem, yet a very hot topic in Artificial Intelligence, NP-hard optimization. The paper demonstrates that $\mathrm{CNN}$ computers outperform conventional digital computers. The second paper studies convergence and stability of the dynamics of the full-range model of CNNs, key in signal processing for real time.

The third paper of this Special Issue by Anton Chernihovskyi et al. shows an example of an application efficiently addressed by the spatial-temporal dynamics of CNN technology: the problem of synchronization phenomena in human electroencephalograms.

This Special Issue includes five papers of CNN implementations on digital platforms. The papers by Zsolt Vörösházi et al. and by Sándor Kocsárdi et al. address FPGA realizations of two different applications: a retina model and simulation of two-dimensional supersonic flows. The paper by Balázs Gergely Soós et al. utilizes a GPU 
for CNN processing. The paper by David Barr and Piotr Dudek deals with the implementation of a software processor array on today, standard microprocessors. Although it does not discuss an actual digital implementation, the paper by Suleyman Malki and Lambert Spaanenburg tackles different strategies to design a $\mathrm{CNN}$-specific integrated processor mainly focusing on the digital domain. All these papers are examples of a general trend of the today CNN technology: using cheap digital and easy programable platforms for the realization of massively fine-grained parallelism.

Nevertheless, when it comes to very demanding or specific applications, the design of more specific and analog or mixed-signal hardware becomes a reality. Moreover, the advent of new technological advances like the CMOS-3D technology or nanodevices will certainly push further the research in this direction. This might be the case of applications like visual attention, tracking, or motion estimation. The last paper of this issue by Jonne Poikonen et al. gives a glimpse of such implementations with the realization of an analog processor array for motion estimation in conventional CMOS technology.

Last, but not least, the guest editors would like to thank the numerous reviewers for their invaluable work during the preparation of this Special Issue as well as all the authors for their effort in submitting their papers. The guest editors would also like to mention that some of the articles on this Special Issue are extended versions of papers presented at 2008 11th International Workshop on Cellular Neural Networks and their Applications, CNNA2008, held in Santiago de Compostela, Spain.

$$
\begin{array}{r}
\text { David López Vilariño } \\
\text { Diego Cabello Ferrer } \\
\text { Victor Manuel Brea Sánchez } \\
\text { Ronald Tetzlaff } \\
\text { Chin-Teng Lin }
\end{array}
$$

\section{References}

[1] L. O. Chua and L. Yang, "Cellular neural networks: theory," IEEE Transactions on Circuits and Systems, vol. 35, no. 10, pp. 1257-1272, 1988. 